\title{
手性导电高分子纳米结构的无手性构筑及手性分离应用
}

刘鸣华

中国科学院化学研究所, 北京 100190

\section{Chiral Polyaniline Nanostructures: Achiral Fabrication and Application for Enantioselective Separation}

\section{Minghua Liu}

Key Laboratory of Colloid, Interface and Chemical Thermodynamics, Chinese Academy of Sciences, Beijing 100190, P. R. China. Emails: liumh@iccas.ac.cn.

Published online: April 13, 2020.

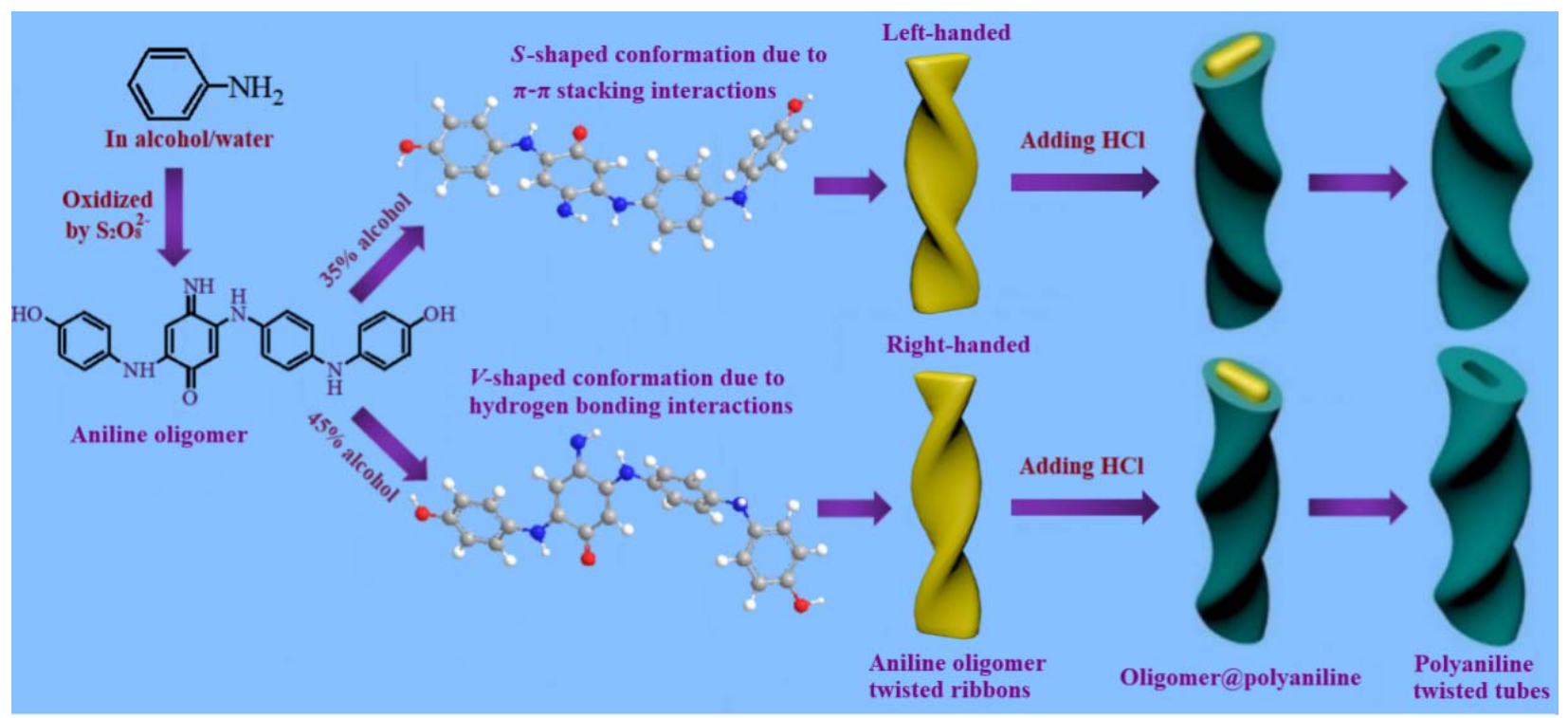

无手性体系中低聚苯胺螺旋纳米带和导电聚苯胺螺旋纳米管的形成。

手性是指两个物体互成镜像、但不能重叠的 特征, 是自然界中普遍存在的现象: 常见的蜗牛壳 是大多是右手螺旋, 而牵牛花的藤大都以右手螺 旋方向缠绕; 在微观分子层面, 比如组成生命体的 一些基本物质, 除甘氨酸以外的氨基酸分子都是 $\mathrm{L}$-型的, 糖分子都是 $\mathrm{D}$-型的。

手性分子能组成宏观手性物体, 但组成宏观 手性物体并不一定需要微观手性物质 ${ }^{1-3}$ 。即非手 性的微观物质(如非手性分子)也可能组装(超分子 组装)成宏观手性物体(超分子结构)。例如, 沙子的 主要成分二氧化硅本身并不是手性分子, 但它可
以堆积成具有手性特征的石英。通常, 这种由非手 性分子组装宏观手性物体的手性是随机的, 有时 得到左旋结构, 有时得到右旋结构, 还有时得到左 旋和右旋结构的混合物 ${ }^{4}$ 。这种由非手性物质形成 手性结构的现象为功能材料手性纳米结构的无手 性构筑提供了可能 5,6 。

作为重要功能性合成材料之一的导电聚苯 胺, 在手性结构的形成以及在手性催化、手性传 感、手性拆分和手性电子器件等领域表现了潜在 的应用价值 ${ }^{7,8}$ 。目前, 导电聚苯胺手性纳米结构一 般在手性掺杂酸或螺旋蛋白模板的诱导下, 以过 
硫酸铵为氧化剂, 在酸性水溶液中化学氧化苯胺 单体进行制备, 且大多数为简单的螺旋纳米带或 纳米纤维。相比之下, 复杂的手性纳米结构(如螺 旋纳米管) 具有可期望的仿生功能, 因而受到更多 的关注。然而, 制备复杂手性纳米结构的功能材料 仍然是具有挑战性的课题。此外, 在无手性体系中 由非手性分子构筑手性纳米结构, 不仅涉及自然 界中手性起源的问题, 而且有助于创造非手性功 能分子的多功能手性材料, 因而具有十分重要的 科学意义和研究价值。

近期, 扬州大学韩杰教授课题组首次在无手 性体系中构筑了手性导电聚苯胺纳米结构并揭示 了导电聚苯胺超分子手性的形成机制 ${ }^{9}$ 。首先, 在 无手性的醇/水混合溶剂中通过苯胺的化学氧化聚 合制备了手性低聚苯胺螺旋纳米带, 螺旋纳米带 的主要成分是含有羟基和羰基的四聚苯胺衍生 物。调节混合溶剂中的醇含量可实现低聚苯胺纳 米带螺旋手性的控制: 比如醇含量为 $35 \%$ 的体系中 可以制备出左旋纳米带, 醇含量为 $45 \%$ 的体系中可 以制备出右旋纳米带。超分子组装机制研究揭示 了 $\pi-\pi$ 堆积和氢键作用的协同是实现超分子手性 组装结构的主要起因。

为进一步提高共轭分子的分子量并优化手性 纳米结构, 韩杰教授课题组在手性低聚苯胺螺旋 纳米带构筑机理和超分子手性组装机制的认识基 础上, 通过调控醇/水混合溶剂的酸度, 借助手性 低聚苯胺螺旋纳米带的活性模板效应构筑了导电 聚苯胺螺旋纳米管 ${ }^{10}$ 。适当酸度的引入, 一方面为 反应初期手性低聚苯胺螺旋纳米带的形成提供保 证, 另一方面, 为低聚苯胺向导电聚苯胺的化学氧 化聚合提供了合适的生长环境。研究发现, 反应初 期生成手性低聚苯胺螺旋纳米带, 随着低聚苯胺 的化学氧化聚合, 低聚苯胺螺旋纳米带作为反应 活性模板导向手性导电聚苯胺螺旋纳米管的生 成。相比手性低聚苯胺螺旋纳米带, 手性电聚苯胺 螺旋纳米管具有更大的比表面积和更好的环境稳 定性。基于导电聚苯胺螺旋纳米管的超分子手性
特征以及导电聚苯胺特殊的酸掺杂特性, 成功实 现了多种氨基酸分子的高效手性拆分, 并展现出 良好的可循环利用性能。与手性低聚苯胺螺旋纳 米带相比, 手性导电聚苯胺螺旋纳米管表现出更 为优越的手性拆分效率。

上述相关研究成果分别在 Journal of the American Chemical Society和ACS Nano 等期刊上 发表 9,10 。相关工作不仅实现了功能聚合物的无手 性制备, 加深了人们对超分子手性组装的理解, 而 且为手性功能材料在手性分离领域的应用提供了 新的思路。

\section{References}

(1) Shen, B.; Kim, Y.; Lee, M. Adv. Mater. 2020, 31, 1905669. doi: 10.1002/adma.201905669

(2) Sang, Y.; Han, J.; Zhao, T.; Duan, P.; Liu, M. Adv. Mater. 2019, 1900110. doi: 10.1002/adma.201900110

(3) Li, Y.; Liu, C.; Bai, X.; Tian, F.; Hu, G.; Sun, J. Angew. Chem. Int. Ed. 2020, 59, 3486. doi: 10.1002/anie.201213882

(4) Sun, J.; Li, Y.; Yan, F.; Liu, C.; Sang, Y.; Tian, F.; Feng, Q.; Duan, P.; Zhang, L.; Shi, X.; et al. Nat. Commun. 2018, 9, 2599. doi: 10.1038/s41467-018-05017-7

(5) Davidsen, J. Nat. Phys. 2018, 14, 207. doi: 10.1038/s41567-017-0014-7

(6) Chen, C.; Kieffer, R.; Ebert, H.; Prehm, M.; Zhang, R. B.; Zeng, X. B.; Liu, F.; Ungar, G.; Tschierske, C. Angew. Chem. Int. Ed. 2020, 59, 2725. doi: 10.1002/anie.201911245

(7) Yang, J.; Liang, J.; Pan, F.; Wang, Z.; Zhang, J.; Amin, K.; Fang, J.; Zou, W.; Chen, Y.; Shi, X.; et al. Nat. Commun. 2018, 9, 3808. doi: 10.1038/s41467-018-06239-5

(8) Shen, Z.; Sang Y.; Wang, T.; Jiang, J.; Meng, Y.; Jiang, Y.; Okuro, K.; Aida, T.; Liu, M. Nat. Commun. 2019, 10, 3976. doi: 10.1038/s41467-019-11840-3

(9) Zhou, C.; Ren, Y.; Han, J.; Gong, X.; Wei, Z.; Xie, J.; Guo, R. J. Am. Chem. Soc. 2018, 140, 9417. doi: 10.1021/jacs.7b12178

(10) Zhou, C.; Ren, Y.; Han, J.; Xu, Q.; Guo, R. ACS Nano 2019, 13, 3534. doi: 10.1021/acsnano.8b09784 